Article

\title{
Corporate Social Responsibility and Corporate Financial Performance: An Empirical Literature Review
}

\author{
Sonia Boukattaya ${ }^{1}$, Zyed Achour $^{2, *}$, Zeineb Hlioui ${ }^{3}$
}

Received: 16 August 2021 / Accepted: 11 September 2021 / Published online: 30 November 2021

\begin{abstract}
This study aims to present a literature review of recent studies on the relationship between environmental, social and governance (ESG) performance, corporate social responsibility (CSR) and corporate financial performance (CFP) and to provide a path for future researches. Using content analysis method, a total of 88 papers published in renowned journals, over the period 2015-2021, were selected in the review. Several findings have been made: first, the majority of researches have focused on the CSR's "social impact" hypothesis on CFP; the reverse relationship seems to have been overlooked. Second, the contested results are likely to be attributable both to differences in research contexts and CSR' laws but also to biases relating to the operationalization of CSR concept and CFP proxies retained. Finally, several arguments are advanced arguing for an indirect link between CSR and CFP. Future research should, therefore, pay attention to the different contingent variables that are likely to affect the studied relationship.
\end{abstract}

Keywords: Literature review, Corporate social responsibility, ESG performance, Firm performance, Firm value

${ }^{1}$ Higher Institute of Management (ISG Tunis), University of Tunis, Tunisia

${ }^{2}$ National Institute of Labor and Social Studies (INTES), University of Carthage, Tunisia

${ }^{3}$ Higher Institute of Management (ISG Tunis), University of Tunis, Tunisia

*Corresponding Email: zyed.achour@intes.rnu.tn

To cite this article:

Boukattaya, S., Achour, Z., \& Hlioui, Z. (2021). Corporate Social Responsibility and Corporate Financial Performance: An Empirical Literature Review. International Journal of Innovative Research and Publications, 1(3), 1-32. https://doi.org/10.51430/IJIRP.2021.13.001 


\section{Introduction}

Facing a series of financial scandals and declining stakeholder confidence, several reforms have been launched worldwide aiming at advancing environmental, social and corporate governance (ESG) issues. The motivation was to move from a shareholder perception of values to a more global perception including the interests of all the stakeholders. As a result, CSR has emerged as a significant dimension for the development of sustainable strategies that affect any given firm performance (Eccles and Serafeim, 2013). In the same way, a large number of academic studies have analyzed the financial implications of CSR. However, the relationship between CSR, firm performance and profitability remains one of the most debated topics in this field since the implications of such a link are not without interest for managers and policymakers but also for shareholders.

CSR-CFP relationship is ambiguous. Although a majority of empirical studies-are in favor of a positive link (Jeong et al. 2018; Chuang and Huang, 2018; Ghaderi et al. 2019; González-Rodríguez et al. 2019; Chung et al. 2018; Kim et al. 2018; Sroufe and GopalakrishnaRemani, 2019), others advocate a negative or non-significant link (Chen et al. 2018; DuqueGrisales and Aguilera-Caracuel, 2019). These heterogeneous results can mainly be explained by the use of different measures of corporate social responsibility and firm performance (Galant and Cadez, 2017), the different studied periods and contexts, the choice of model specification and control variables (Benlemlih, 2017). Recent studies have deepened the understanding by investigating the channels through which the CSR impacts the firm value. Moreover, there is a growing interest in studying this relationship by industry and to study the impact of individual CSR components effects on firm value.

The main goal of this paper was to review the recent empirical literature focused on the impact of corporate social responsibility on firm value and profitability. Thus, its main objective was to provide a better understanding of the recent literature and help to identify how CSR affects firm performance. We provided an overview of the current state of research and suggested a future research scope in this field for the help of both academicians and practitioners.

The remainder of this paper is structured as follows. In the second section, we explained the adapted methodology in this literature review. In section three, we described the theories affecting the relationship between CSR and firm performance. In sections four and five, we introduced the different metrics of profitability, firm value and CSR. In section six, we 
reviewed the empirical results before drawing the main conclusions of this review in the last section.

\section{Review Methodology}

The literature search was conducted in two steps. In the first, we began with a random search of articles with the help of various search items. We looked for search items in articles' titles, abstracts or keywords. Articles including the following keywords i.e. corporate social responsibility, corporate social performance, CSR, ESG, environmental performance, social performance, corporate sustainability together with profitability, corporate performance, corporate financial performance, firm value (and synonyms) published during the period January 2015 to August 2021 were included to the selection

To ensure the quality of the included studies in the review, we selected the articles, papers and chapters published by the following academic known publishers i.e. Springer, SAGE, Emerald, Inderscience, Wiley, MDPI, Routledge and Elsevier, in the second step. Almost all the studies are from journals which are available on the following indices i.e. Scopus Index, Australian Business Deans Council (ABDC) master list, Journal Citation Index of Web of Science. The above two steps allowed obtaining 88 empirical studies related to the corporate social responsibility, firm value and profitability relationship. Table 1 summarizes CSR, firm profitability and value metrics and the main conclusions of the selected papers.

\section{CSR, Firm Value and Profitability Link: Theoretical Basis}

Three theories have studied the relationship between social and financial performance of companies. The first, the agency theory suggests that social practices only make sense if they increase the shareholders' profit, considering that these practices only entail costs causing competitive disadvantages for the company. According to the agency theory (Jensen and Meckling, 1976), the objective of a company is to increase the wealth of shareholders. Friedman (1962) sums up this argument better by asserting that maximizing shareholders' profits is the sole social responsibility of the company. He identifies CSR as an agency problem in which managers misappropriate the wealth of shareholders to pursue a social mission of their choice. In addition, Friedman (1970) suggests that environmental or social responsibility activities that exceed the minimum legal standards would cause additional costs and therefore reduce the firm value. In fact, the use of resources for social spending places companies at a competitive disadvantage vis-à-vis competitors whose managers do not pursue a social mission 
other than maximizing the shareholder value (Friedman, 1970). The agency model supports the hypothesis that a company engaging in low CSR is more efficient and profitable. In fact, it would not incur the same financial expenses as a company that invests in several forms of social responsibility, such as employee well-being, environmental protection and charitable donations.

From the agency theory perspective, a socially responsible investing may waste resources that can weaken, rather than strengthen, a business, with the shareholders' loss of wealth. Opting for investing in CSR is a decision based on principles that expose the company to a risk in relation to the objective of maximizing financial value (Jensen, 2001). Consequently, the firm is more exposed to the opportunistic behavior of managers who choose to overinvest in CSR so as to improve their image and their social legitimacy. For example, if a firm invests in equipment that reduces pollution to a greater degree than that required by law, the costs of this equipment may exceed the financial benefits granted to the investors. This situation goes against the good management practices.

The second, the stakeholders' theory claims that social responsibility is a duty for the company through which it improves the satisfaction of all stakeholders and therefore the economic and financial performance of the company. From the stakeholders' theory perspective, companies depend on stakeholders to survive since they provide the essential tangible or intangible resources necessary for their success (Hill and Jones, 1992). If stakeholders withdraw resources in whole or in part, a business may not be able to continue operating or endure financial disaster (Clarkson, 1995; Harjoto et al. 2015). The concept of stakeholders has been defined as "any group or individual who can affect or is affected by the achievement of the goals of an organization. In the broad sense, the term includes suppliers, customers, shareholders, employees, communities, political authorities (national and territorial), the media, etc. (Freeman, 1984). According to Freeman (1984), the stakeholder theory identifies the generation of value as a central engine of business, but similarly asserts that this value must be shared by a group of stakeholders that includes not only shareholders and managers but also all the actors who may have an interest in the activity of the company. This is the theoretical framework currently used by most research on social responsibility.

The positive effect of social responsibility on financial performance is best explained by the social impact hypothesis, which is itself based on the theory of stakeholders (Freeman, 1984). This hypothesis suggests that by meeting the demands and expectations of different stakeholders, companies are reforming their financial performance (Perrini et al. 2011). CSR 
is considered a strategic tool for obtaining financial advantages by means of competitive advantages on the market (Porter and Kramer, 2002), an improved reputation (Fombrun and Shanley, 1990), a better brand image (Murray and Montanari , 1986) and more legitimacy (Hart and Christensen, 2002). For Cornell and Shapiro (1987), a company which ignores the preferences of interest groups will suffer a negative effect on its own reputation, thereby increasing its risk premium and, consequently, reducing its profitability. Regarding this aspect, Cornell and Shapiro (1987) argue that the cost of CSR is almost negligible compared to its potential benefits.

The third, the Resource-Based View (RBV) theory (Barney, 1991), provides enough arguments to support the hypothesis of creating competitive advantage from the exercise of CSR (Barney, 1991). This approach deals with the link between the internal characteristics of the company and its performance. Indeed, according to this theory, the performance differentials are mainly explained by the existence of company-specific resources supposed to be precious, scarce, difficult to imitate by rivals and not easily tradable on the markets (therefore hardly substitutable). For Barney, (1991); Bowman and Ambrosini, (2003), these resources must be "ambiguous" that is to say that the processes of their creation, are not completely known; they are also "complex" insofar as certain resources such as reputation, corporate culture are difficult to change in the short term, as they are "inimitable".

Furthermore, the resource-based approach defends the idea that CSR is used strategically by companies to create and consolidate a sustainable competitive advantage (McWilliams and Siegel, 2001; Zhao and al. 2019). Indeed, this approach suggests that CSR is one of the heterogeneous resources and skills whose mobility between firms remains imperfect, especially if we consider that these resources remain scarce, inimitable and non-substitutable. Thus, CSR and its perception by the different stakeholders can constitute a sustainable competitive advantage, a kind of "intangible" capital. For example, McWilliams and Siegel (2001) have shown that the addition of a social attribute to a product has an impact on demand and subsequently on the profitability of the company. Werner (2009) argues that CSR as an "intangible" resource is increasingly integrated into the various business processes and that, when well designed and adapted to the needs of the community and society, it may become a source of opportunities, innovation and a competitive advantage for companies.

The previous discussion suggests that corporate engagement in CSR could serve as a control mechanism to balance the interest of multiple stakeholder groups (Mason and Simmons, 2014). In addition, the satisfaction of all stakeholders tends to increase the reputation 
of the company, which in turn has a positive impact on financial performance. Conversely, disappointment of stakeholders may have a negative impact on financial performance. This theoretical framework is at the center of the strategic management given the development of CSR and sustainability concepts that make the company better take into account the consequences of their activities on all stakeholders on the one hand, and the environment, on the other (Donaldson and Preston, 1995).

Table 1. An overview of the recent studies on the relationship between CSR and firm value and profitability discussed in this paper

\begin{tabular}{|c|c|c|c|c|c|c|c|}
\hline \multirow[t]{2}{*}{ Authors } & \multirow[t]{2}{*}{ Market } & \multirow{2}{*}{$\begin{array}{c}\text { CSR } \\
\text { Measure }\end{array}$} & \multirow{2}{*}{$\begin{array}{c}\text { Firm value } \\
\text { and } \\
\text { Profitability } \\
\text { measures }\end{array}$} & \multicolumn{4}{|c|}{ Results } \\
\hline & & & & + & - & $\mathbf{0}$ & $\begin{array}{l}\text { Mediator/ } \\
\text { moderator }\end{array}$ \\
\hline $\begin{array}{l}\text { Bai and } \\
\text { Chang (2015) }\end{array}$ & $\begin{array}{l}\text { Internation } \\
\text { al }\end{array}$ & $\begin{array}{l}\text { CSR scores } \\
\text { developed by } \\
\text { authors }\end{array}$ & $\begin{array}{l}\text {-Growth rate } \\
\text {-Returns on } \\
\text { investment } \\
\text { (ROI) }\end{array}$ & $\sqrt{ }$ & & & $\begin{array}{l}\text { Marketing } \\
\text { competence }\end{array}$ \\
\hline $\begin{array}{l}\text { Saeidi et al. } \\
(2015)\end{array}$ & Iran & $\begin{array}{l}\text { CSR score } \\
\text { developed by } \\
\text { authors from } \\
\text { annual } \\
\text { reports }\end{array}$ & $\begin{array}{l}\text {-Sales growth } \\
\text {-ROE } \\
\text {-ROS } \\
\text {-ROA } \\
\text {-ROI } \\
\text {-Net profit } \\
\text { margin of the } \\
\text { firm }\end{array}$ & $\sqrt{ }$ & & & $\begin{array}{l}\text {-Reputation } \\
\text {-Competitive } \\
\text { advantage }\end{array}$ \\
\hline $\begin{array}{l}\text { Wang et al. } \\
(2015)\end{array}$ & China & $\begin{array}{l}\text { Economic, } \\
\text { Environment } \\
\text { al, Social and } \\
\text { Governance } \\
\text { index } \\
\text { developed by } \\
\text { authors using } \\
\text { the } \\
\text { conceptual } \\
\text { scheme of } \\
\text { Dow Jones } \\
\text { Sustainability } \\
\text { Index }\end{array}$ & $\begin{array}{l}\text {-ROA } \\
\text {-ROE } \\
\text {-Tobin's Q }\end{array}$ & $\sqrt{ }$ & & & \\
\hline $\begin{array}{l}\text { Youn et al. } \\
(2015)\end{array}$ & U.S & $\begin{array}{l}\text { CSR score, } \\
\text { CSR strength } \\
\text { score and } \\
\text { CSR } \\
\text { concerns } \\
\text { score from }\end{array}$ & Tobin's Q & $\sqrt{ }$ & & & Firm size \\
\hline
\end{tabular}




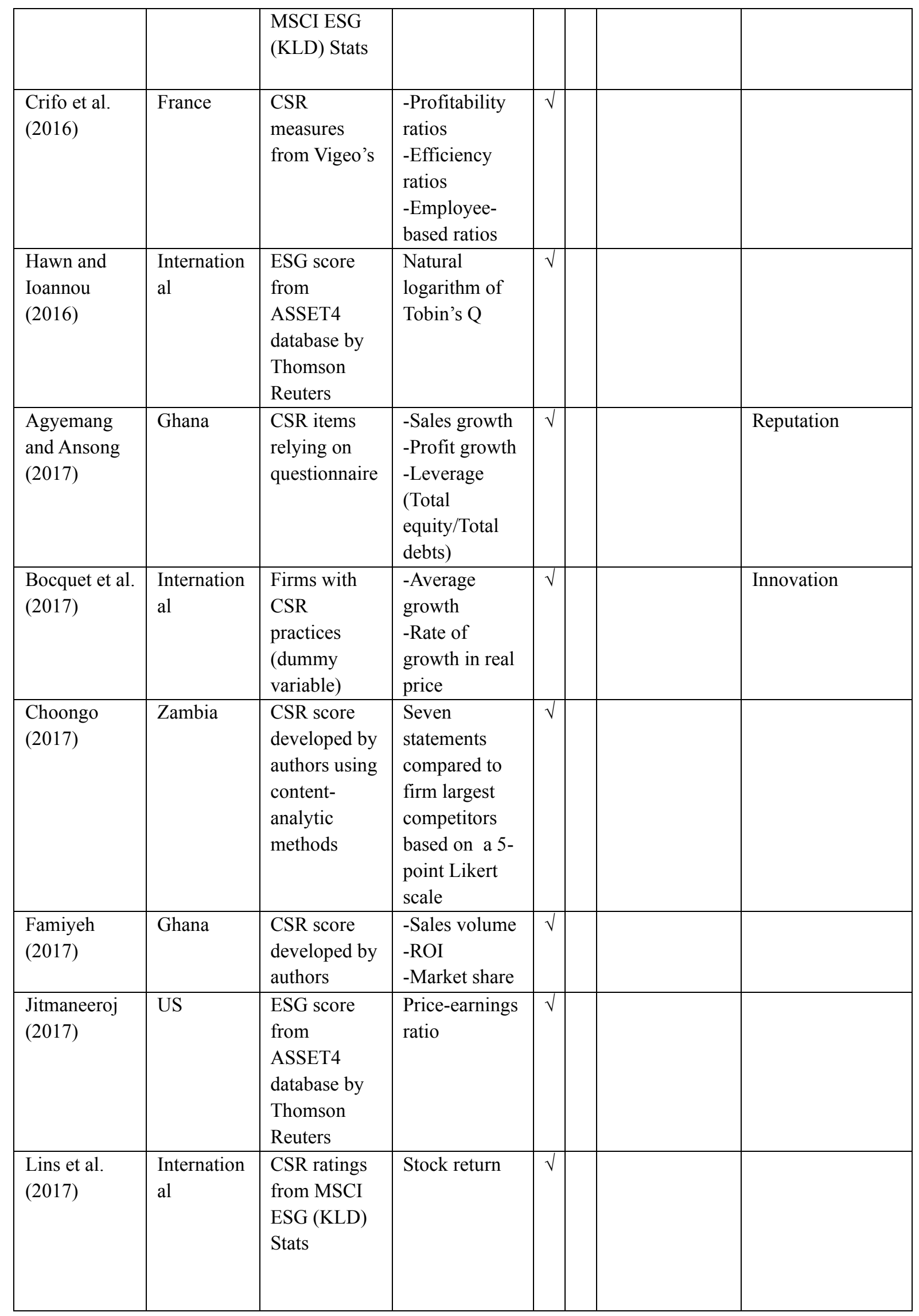




\begin{tabular}{|c|c|c|c|c|c|c|}
\hline $\begin{array}{l}\text { Martinez- } \\
\text { Conesa et al. } \\
\text { (2017) }\end{array}$ & Spain & $\begin{array}{l}\text {-CSR with } \\
\text { employees } \\
\text {-CSR with } \\
\text { customers } \\
\text {-CSR with } \\
\text { suppliers } \\
\text {-CSR with } \\
\text { local } \\
\text { community } \\
\text { From CSA } \\
\text { Consultants } \\
\text { Company }\end{array}$ & $\begin{array}{l}\text {-Profits } \\
\text { growth in the } \\
\text { last } 3 \text { years } \\
\text {-Return on } \\
\text { assets growth } \\
\text { in the last } 3 \\
\text { years }\end{array}$ & $\sqrt{ }$ & & $\begin{array}{l}\text { Innovation } \\
\text { performanc } \\
\text { e }\end{array}$ \\
\hline $\begin{array}{l}\text { Nekhili et al. } \\
\text { (2017) }\end{array}$ & France & $\begin{array}{l}\text { CSR } \\
\text { disclosure } \\
\text { index } \\
\text { developed by } \\
\text { authors from } \\
\text { annual } \\
\text { reports }\end{array}$ & Tobin's Q & $\sqrt{ }$ & & $\begin{array}{l}\text { Family } \\
\text { involvemen } \\
t\end{array}$ \\
\hline $\begin{array}{l}\text { Price and } \\
\text { Sun (2017) }\end{array}$ & U.S & $\begin{array}{l}\text { CSR } \\
\text { strengths and } \\
\text { CSR } \\
\text { concerns } \\
\text { from MSCI } \\
\text { ESG (KLD) } \\
\text { Stats }\end{array}$ & Tobin's Q & $\sqrt{ }$ & & \\
\hline Velte (2017) & Germany & $\begin{array}{l}\text { Aggregate } \\
\text { ESG score } \\
\text { and } \\
\text { dimensions } \\
\text { of ESG score } \\
\text { from } \\
\text { ASSET4 } \\
\text { database by } \\
\text { Thomson } \\
\text { Reuters }\end{array}$ & $\begin{array}{l}\text {-Tobin's Q } \\
\text {-ROA }\end{array}$ & $\sqrt{ }$ & $\sqrt{ }$ & \\
\hline $\begin{array}{l}\text { Anser et al. } \\
\text { (2018) }\end{array}$ & Pakistan & $\begin{array}{l}\text { CSR score } \\
\text { developed by } \\
\text { authors using } \\
\text { content- } \\
\text { analytic } \\
\text { methods }\end{array}$ & ROA & $\sqrt{ }$ & & \\
\hline $\begin{array}{l}\text { Aouadi and } \\
\text { Marsat } \\
(2018)\end{array}$ & $\begin{array}{l}\text { Internation } \\
\text { al }\end{array}$ & $\begin{array}{l}\text { ESG score } \\
\text { from } \\
\text { ASSET4 } \\
\text { database by } \\
\text { Thomson } \\
\text { Reuters }\end{array}$ & Tobin's Q & $\sqrt{ }$ & & \\
\hline
\end{tabular}




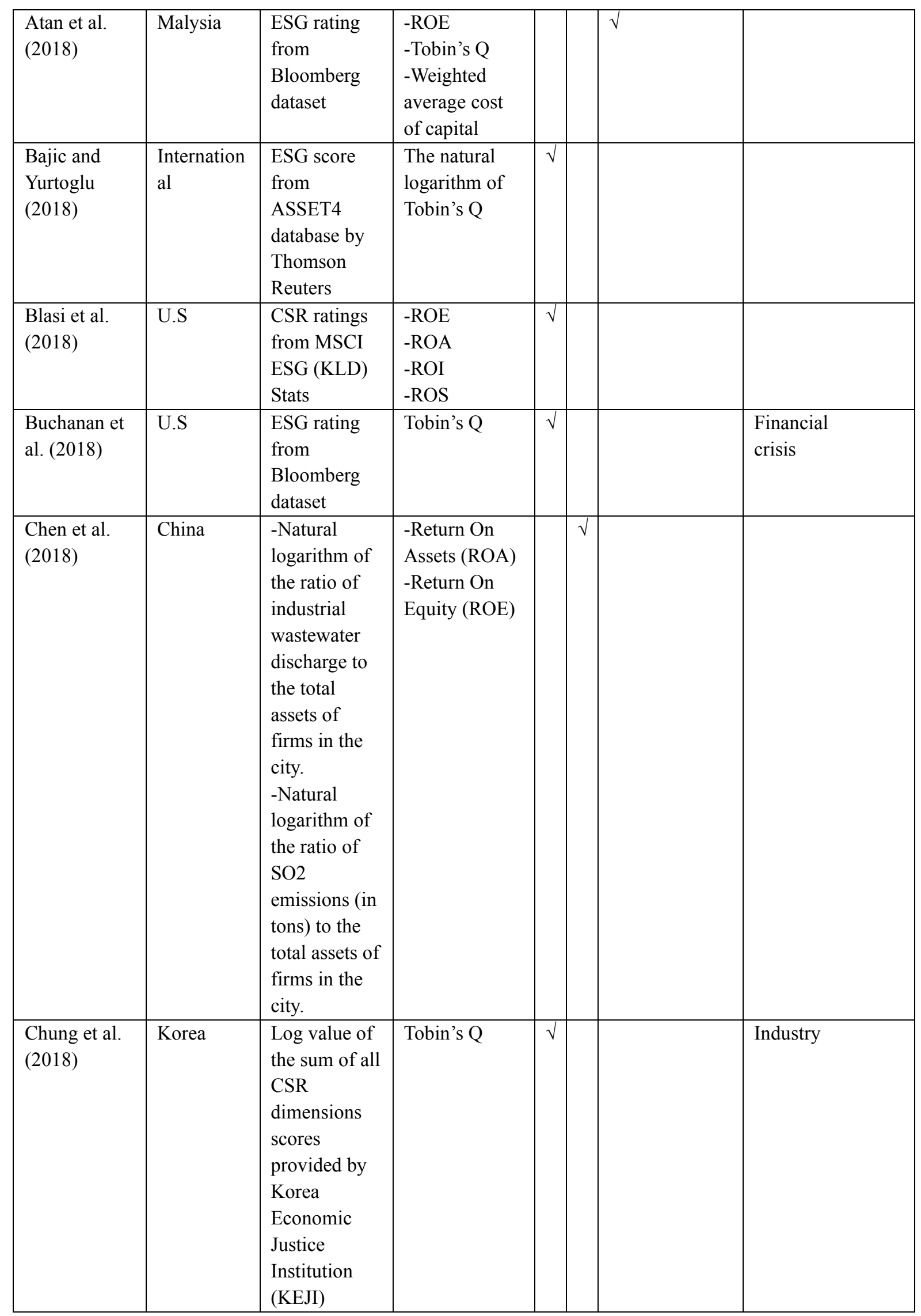




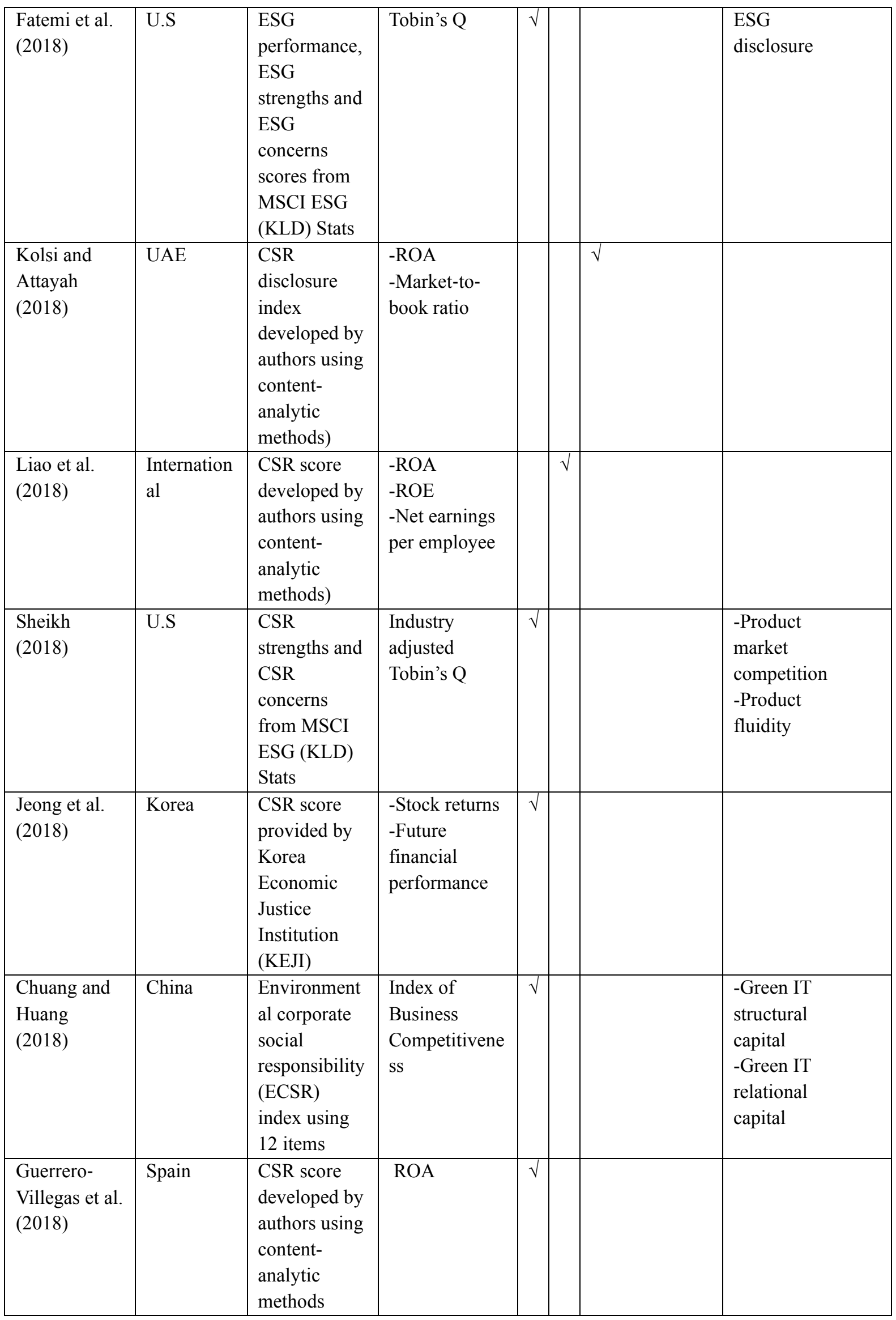




\begin{tabular}{|c|c|c|c|c|c|}
\hline $\begin{array}{l}\text { Harjoto and } \\
\text { Laksmana } \\
(2018)\end{array}$ & U.S & $\begin{array}{l}\text { CSR ratings } \\
\text { from MSCI } \\
\text { ESG (KLD) } \\
\text { Stats }\end{array}$ & Tobin's Q & $\sqrt{ }$ & \\
\hline $\begin{array}{l}\text { Hasan et al. } \\
\text { (2018) }\end{array}$ & $\begin{array}{l}\text { U.S. } \\
(1992- \\
2009)\end{array}$ & $\begin{array}{l}\text { CSR ratings } \\
\text { from MSCI } \\
\text { ESG (KLD) } \\
\text { Stats }\end{array}$ & Tobin's Q & $\sqrt{ }$ & $\begin{array}{l}\text { Total factor } \\
\text { productivity }\end{array}$ \\
\hline $\begin{array}{l}\text { Kao et al. } \\
\text { (2018) }\end{array}$ & China & $\begin{array}{l}\text { CSR rating } \\
\text { from the } \\
\text { China CSR } \\
\text { Research } \\
\text { Center }\end{array}$ & Tobin's Q & $\sqrt{ }$ & $\begin{array}{l}\text { Firm } \\
\text { ownership } \\
\text { type. }\end{array}$ \\
\hline $\begin{array}{l}\text { Kim et al. } \\
\text { (2018) }\end{array}$ & U.S & $\begin{array}{l}\text { CSR } \\
\text { strengths and } \\
\text { CSR } \\
\text { concerns } \\
\text { from MSCI } \\
\text { ESG (KLD) } \\
\text { Stats }\end{array}$ & Tobin's Q & $\sqrt{ }$ & $\begin{array}{l}\text { Firm's } \\
\text { competitive } \\
\text {-action } \\
\text { level }\end{array}$ \\
\hline $\begin{array}{l}\text { Maqbool and } \\
\text { Zameer } \\
(2018)\end{array}$ & India & $\begin{array}{l}\text { CSR score } \\
\text { developed by } \\
\text { authors using } \\
\text { content- } \\
\text { analytic } \\
\text { methods }\end{array}$ & $\begin{array}{l}\text {-ROE } \\
\text {-ROA } \\
\text {-Net Profit } \\
\text {-Stock return } \\
\text {-Private equity }\end{array}$ & $\sqrt{ }$ & \\
\hline $\begin{array}{l}\text { Miller et al. } \\
(2018)\end{array}$ & U.S & $\begin{array}{l}\text { A dummy } \\
\text { variable that } \\
\text { equals one if } \\
\text { a bank moves } \\
\text { from a non- } \\
\text { compliant } \\
\text { with } \\
\text { Community } \\
\text { Reinvestment } \\
\text { Act (CRA) } \\
\text { rating in the } \\
\text { prior period } \\
\text { to a } \\
\text { satisfactory } \\
\text { CRA rating } \\
\text { in the next } \\
\text { period, zero } \\
\text { otherwise. }\end{array}$ & ROA & $\sqrt{ }$ & \\
\hline $\begin{array}{l}\text { Ramanathan } \\
\text { (2018) }\end{array}$ & UK. & $\begin{array}{l}- \\
\text { Environment } \\
\text { al } \\
\text { certifications } \\
\text {-Self- } \\
\text { evaluation of } \\
\text { environmenta }\end{array}$ & $\begin{array}{l}\text {-Sales growth } \\
\text {-Improvement } \\
\text { in market } \\
\text { share. }\end{array}$ & $\sqrt{ }$ & \\
\hline
\end{tabular}




\begin{tabular}{|c|c|c|c|c|c|c|}
\hline & & $\begin{array}{l}1 \\
\text { performance } \\
\text { over the past } \\
5 \text { years. }\end{array}$ & & & & \\
\hline $\begin{array}{l}\text { Rasheed et } \\
\text { al. (2018) }\end{array}$ & Pakistan & $\begin{array}{l}\text {-Sum of } \\
\text { spending on } \\
\text { environment, } \\
\text { social } \\
\text { activities, } \\
\text { employees } \\
\text { welfare and } \\
\text { philanthropy } \\
\text {-Total CSR } \\
\text { words in an } \\
\text { annual report }\end{array}$ & $\begin{array}{l}\text {-Earnings per } \\
\text { share } \\
\text {-Profit before } \\
\text { tax } \\
\text {-Return on } \\
\text { sales } \\
\text {-Return on } \\
\text { equity } \\
\text {-Return on } \\
\text { assets }\end{array}$ & $\sqrt{ }$ & & \\
\hline $\begin{array}{l}\text { Sial et al. } \\
(2018, a)\end{array}$ & China & $\begin{array}{l}11 \text { items } \\
\text { measure } \\
\text { referring to } \\
\text { GRI } \\
\text { Sustainability } \\
\text { Reporting } \\
\text { Guideline or } \\
\text { Not }\end{array}$ & Tobin's Q & $\sqrt{ }$ & & \\
\hline $\begin{array}{l}\text { Sial et al. } \\
(2018, b)\end{array}$ & China & $\begin{array}{l}\text { CSR ratings } \\
\text { provided by } \\
\text { Rankins } \\
(\mathrm{RKS})\end{array}$ & Tobin's Q & $\sqrt{ }$ & & $\begin{array}{l}\text { Earnings } \\
\text { managemen } \\
\mathrm{t}\end{array}$ \\
\hline $\begin{array}{l}\text { Yoon et al. } \\
(2018)\end{array}$ & Korea & $\begin{array}{l}\text { ESG scores } \\
\text { from the } \\
\text { Korean } \\
\text { Corporate } \\
\text { Governance } \\
\text { Service } \\
\text { (KCGS) }\end{array}$ & $\begin{array}{l}\text {-Book Value } \\
\text { per share } \\
\text {-Earning per } \\
\text { share }\end{array}$ & $\sqrt{ }$ & & \\
\hline $\begin{array}{l}\text { Yu et al. } \\
(2018)\end{array}$ & $\begin{array}{l}\text { Internation } \\
\text { al }\end{array}$ & $\begin{array}{l}\text { ESG } \\
\text { disclosure } \\
\text { rating from } \\
\text { Bloomberg } \\
\text { dataset }\end{array}$ & Tobin's Q & $\sqrt{ }$ & & \\
\hline $\begin{array}{l}\text { Akben- } \\
\text { Selcuk } \\
(2019)\end{array}$ & Turkey & $\begin{array}{l}\text { CSR takes } \\
\text { the value of } 1 \\
\text { if firm is in } \\
\text { the Borsa } \\
\text { Istanbul } \\
\text { Sustainability } \\
\text { Index and } 0 \\
\text { otherwise }\end{array}$ & $\mathrm{ROA}$ & $\sqrt{ }$ & & $\begin{array}{l}\text { ownership } \\
\text { concentratio } \\
\mathrm{n}\end{array}$ \\
\hline $\begin{array}{l}\text { Al-Hadi et al. } \\
(2019)\end{array}$ & Australia & $\begin{array}{l}75 \text { items of } \\
\text { CSR activity }\end{array}$ & $\begin{array}{l}\text { Financial } \\
\text { distress }\end{array}$ & & $\sqrt{ }$ & $\begin{array}{l}\text { Mature life } \\
\text { cycle stages }\end{array}$ \\
\hline
\end{tabular}




\begin{tabular}{|c|c|c|c|c|c|c|}
\hline & & $\begin{array}{l}\text { based on } \\
\text { Global } \\
\text { Reporting } \\
\text { Initiative } \\
\text { (GRI) G4 } \\
\text { sustainability } \\
\text { reporting } \\
\text { guidelines }\end{array}$ & $\begin{array}{l}\text { measured by } \\
\text { models in } \\
\text { previous } \\
\text { literature }\end{array}$ & & & \\
\hline $\begin{array}{l}\text { Bing and Li } \\
\text { (2019) }\end{array}$ & China & $\begin{array}{l}\text { CSR rating } \\
\text { from } \\
\text { Bloomberg } \\
\text { dataset }\end{array}$ & Tobin Q & & $\sqrt{ }$ & \\
\hline $\begin{array}{l}\text { Broadstock et } \\
\text { al. (2019) }\end{array}$ & Japan & $\begin{array}{l}\text { ESG score } \\
\text { from MSCI } \\
\text { ESG (KLD) } \\
\text { Stats }\end{array}$ & $\begin{array}{l}\text {-ROA } \\
\text {-ROE } \\
\text {-Tobin's Q }\end{array}$ & $\sqrt{ }$ & & \\
\hline $\begin{array}{l}\text { Cao et al. } \\
(2019)\end{array}$ & U.S & $\begin{array}{l}\text { Economic, } \\
\text { Environment } \\
\text { al and Social } \\
\text { scores } \\
\text { developed by } \\
\text { authors }\end{array}$ & $\begin{array}{l}\text {-Abnormal- } \\
\text { return of the } \\
\text { stock price } \\
\text {-Idiosyncratic- } \\
\text { risk of the } \\
\text { stock price }\end{array}$ & & $\sqrt{ }$ & \\
\hline $\begin{array}{l}\text { Chang et al. } \\
\text { (2019) }\end{array}$ & $\begin{array}{l}\text { Internation } \\
\text { al }\end{array}$ & $\begin{array}{l}\text { ESG score } \\
\text { ASSET } 4\end{array}$ & Tobin's Q & $\sqrt{ }$ & & $\begin{array}{l}\text { Media } \\
\text { freedom }\end{array}$ \\
\hline $\begin{array}{l}\text { Cherian et al. } \\
(2019)\end{array}$ & India & $\begin{array}{l}\text { CSR score } \\
\text { developed by } \\
\text { authors using } \\
\text { content- } \\
\text { analytic } \\
\text { methods }\end{array}$ & $\begin{array}{l}\text {-ROA } \\
\text {-ROE } \\
\text {-Return on } \\
\text { capital } \\
\text { employed } \\
\text {-Profit before } \\
\text { taxation } \\
\text {-Market to } \\
\text { book value } \\
\text { (MTBV) } \\
\text {-Leverage } \\
\text { Turnover }\end{array}$ & $\sqrt{ }$ & & \\
\hline $\begin{array}{l}\text { Duque- } \\
\text { Grisales and } \\
\text { Aguilera- } \\
\text { Caracuel } \\
(2019)\end{array}$ & $\begin{array}{l}\text { Internation } \\
\text { al }\end{array}$ & $\begin{array}{l}\text { ESG score } \\
\text { from } \\
\text { ASSET4 } \\
\text { database by } \\
\text { Thomson } \\
\text { Reuters }\end{array}$ & $\mathrm{ROA}$ & & $\sqrt{ }$ & $\begin{array}{l}\text {-Financial } \\
\text { slack } \\
- \\
\text { Geographic } \\
\text { internationa } \\
1 \\
\text { diversificati } \\
\text { on }\end{array}$ \\
\hline $\begin{array}{l}\text { Garcia et al. } \\
(2019)\end{array}$ & $\begin{array}{l}\text { Internation } \\
\text { al }\end{array}$ & $\begin{array}{l}\text { ESG score } \\
\text { from } \\
\text { ASSET4 } \\
\text { database by }\end{array}$ & $\begin{array}{l}\text {-ROA } \\
\text {-Free cash } \\
\text { flow } \\
\text {-Market } \\
\text { capitalization }\end{array}$ & $\sqrt{ }$ & & \\
\hline
\end{tabular}




\begin{tabular}{|c|c|c|c|c|c|c|}
\hline & & $\begin{array}{l}\text { Thomson } \\
\text { Reuters }\end{array}$ & & & & \\
\hline $\begin{array}{l}\text { García- } \\
\text { Sánchez and } \\
\text { Martínez- } \\
\text { Ferrero } \\
(2019)\end{array}$ & $\begin{array}{l}\text { Internation } \\
\text { al }\end{array}$ & $\begin{array}{l}\text { Strength and } \\
\text { concern } \\
\text { ratings from } \\
\text { EIRIS } \\
\text { database }\end{array}$ & Tobin's Q & $\sqrt{ }$ & & CEO ability \\
\hline $\begin{array}{l}\text { Ghaderi et al. } \\
\text { (2019) }\end{array}$ & Iran & $\begin{array}{l}\text { CSR items } \\
\text { relying on } \\
\text { questionnaire }\end{array}$ & Questionnaire & $\sqrt{ }$ & & \\
\hline $\begin{array}{l}\text { González- } \\
\text { Rodríguez et } \\
\text { al. (2019) }\end{array}$ & Spain. & $\begin{array}{l}\text { Questionnair } \\
\text { e to the hotel } \\
\text { general } \\
\text { managers } \\
\text { related to } \\
\text { four } \\
\text { dimensions } \\
\text { of CSR: } \\
\text { Environment } \\
\text { al, local } \\
\text { community, } \\
\text { customer, and } \\
\text { labor }\end{array}$ & $\begin{array}{l}\text {-Profit margin } \\
\text {-Revenue per } \\
\text { available room } \\
\text {-Net profit }\end{array}$ & & & Reputation \\
\hline Hou (2019) & Taiwan & $\begin{array}{l}\text { CSR awards } \\
\text { as a social } \\
\text { responsibility } \\
\text { indicator }\end{array}$ & Tobin's Q & $\sqrt{ }$ & & \\
\hline $\begin{array}{l}\text { Landi and } \\
\text { Sciarelli } \\
(2019)\end{array}$ & Italy & $\begin{array}{l}\text { ESG ratings } \\
\text { issued by } \\
\text { Standard } \\
\text { Ethics }\end{array}$ & $\begin{array}{l}\text { Abnormal } \\
\text { returns }\end{array}$ & & $\sqrt{ }$ & \\
\hline $\begin{array}{l}\text { Murwaningsa } \\
\text { ri (2019) }\end{array}$ & Indonesia & $\begin{array}{l}\text { Statements of } \\
\text { corporate } \\
\text { social } \\
\text { responsibility } \\
\text { disclosure } \\
\text { from annual } \\
\text { reports }\end{array}$ & Tobin's Q & $\sqrt{ }$ & & \\
\hline $\begin{array}{l}\text { Nekhili et al. } \\
\text { (2019) }\end{array}$ & France & $\begin{array}{l}\text { ESG score } \\
\text { from } \\
\text { ASSET4 } \\
\text { database by } \\
\text { Thomson } \\
\text { Reuters }\end{array}$ & Tobin's Q & $\sqrt{ }$ & & \\
\hline
\end{tabular}




\begin{tabular}{|c|c|c|c|c|c|c|}
\hline $\begin{array}{l}\text { Sekhon } \\
\text { and Kathuria } \\
(2019)\end{array}$ & India & $\begin{array}{l}\text { CSR score } \\
\text { developed by } \\
\text { authors using } \\
\text { content- } \\
\text { analytic } \\
\text { methods }\end{array}$ & $\begin{array}{l}\text {-ROA } \\
\text {-ROE } \\
\text {-Net Profit } \\
\text { Margin }\end{array}$ & & $\sqrt{ }$ & \\
\hline $\begin{array}{l}\text { Siueia et al. } \\
\text { (2019) }\end{array}$ & $\begin{array}{l}\text { Republic } \\
\text { of South } \\
\text { Africa and } \\
\text { Mozanbiqu } \\
\text { e }\end{array}$ & $\begin{array}{l}\text { CSR score } \\
\text { developed by } \\
\text { authors using } \\
\text { content- } \\
\text { analytic } \\
\text { methods }\end{array}$ & $\begin{array}{l}\text { ROA } \\
-\mathrm{ROE}\end{array}$ & & $\sqrt{ }$ & \\
\hline $\begin{array}{l}\text { Sroufe and } \\
\text { Gopalakrishn } \\
\text { a-Remani } \\
(2019)\end{array}$ & U.S & $\begin{array}{l}\text { ESG } \\
\text { disclosure } \\
\text { score from } \\
\text { Bloomberg } \\
\text { dataset }\end{array}$ & $\begin{array}{l}\text {-ROI } \\
\text {-ROA } \\
\text {-Net profit } \\
\text { margin }\end{array}$ & $\sqrt{ }$ & & \\
\hline Velte (2019) & Germany & $\begin{array}{l}\text { ESG } \\
\text { performance } \\
\text { (in total and } \\
\text { its three } \\
\text { pillars) from } \\
\text { from } \\
\text { ASSET4 } \\
\text { database by } \\
\text { Thomson } \\
\text { Reuters }\end{array}$ & ROA & $\sqrt{ }$ & & CEO power. \\
\hline $\begin{array}{l}\text { Walker et al. } \\
\text { (2019) }\end{array}$ & $\begin{array}{l}\text { Internation } \\
\text { al }\end{array}$ & $\begin{array}{l}\text { CSR } \\
\text { strengths and } \\
\text { CSR } \\
\text { concerns } \\
\text { from } \\
\text { Sustainalytics } \\
\text { ratings }\end{array}$ & Tobin's Q & $\sqrt{ }$ & & $\begin{array}{l}\text { Institutional } \\
\text { environmen } \\
t\end{array}$ \\
\hline $\begin{array}{l}\text { Zhao et al. } \\
\text { (2019) }\end{array}$ & China & $\begin{array}{l}\text { ESG score } \\
\text { developed by } \\
\text { authors from } \\
\text { annual } \\
\text { reports }\end{array}$ & $\begin{array}{l}\text { Return on } \\
\text { capital } \\
\text { employed }\end{array}$ & $\sqrt{ }$ & & \\
\hline $\begin{array}{l}\text { Zolotoy et al. } \\
\text { (2019) }\end{array}$ & US & $\begin{array}{l}\text { ESG score } \\
\text { from MSCI } \\
\text { ESG (KLD) } \\
\text { Stats }\end{array}$ & Tobin's Q & $\sqrt{ }$ & & $\begin{array}{l}\text { local } \\
\text { religious } \\
\text { norms }\end{array}$ \\
\hline $\begin{array}{l}\text { Awaysheh et } \\
\text { al. (2020) }\end{array}$ & US & $\begin{array}{l}\text { CSR score } \\
\text { from MSCI } \\
\text { ESG (KLD) } \\
\text { Stats }\end{array}$ & Tobin's Q & $\sqrt{ }$ & & \\
\hline $\begin{array}{l}\text { Bardos et al. } \\
(2020)\end{array}$ & US & $\begin{array}{l}\text { CSR score } \\
\text { from MSCI }\end{array}$ & ROA & $\sqrt{ }$ & & $\begin{array}{l}\text { product } \\
\text { market } \\
\text { perception }\end{array}$ \\
\hline
\end{tabular}




\begin{tabular}{|c|c|c|c|c|c|c|}
\hline & & $\begin{array}{l}\text { ESG (KLD) } \\
\text { Stats }\end{array}$ & & & & \\
\hline $\begin{array}{l}\text { D'Amato and } \\
\text { Falivena } \\
(2020)\end{array}$ & $\begin{array}{l}\text { Western } \\
\text { Europe }\end{array}$ & $\begin{array}{l}\text { ESG score } \\
\text { from MSCI } \\
\text { ESG (KLD) } \\
\text { Stats }\end{array}$ & Tobin's Q & $\sqrt{ }$ & & $\begin{array}{l}\text { firm size } \\
\text { firm age }\end{array}$ \\
\hline $\begin{array}{l}\text { Franco et al. } \\
(2020)\end{array}$ & $\begin{array}{l}\text { Internation } \\
\text { al }\end{array}$ & ESG score & ROE & $\sqrt{ }$ & $\begin{array}{l}\text { U- } \\
\text { shaped }\end{array}$ & $\begin{array}{l}\text { Quality } \\
\text { managemen } \\
\mathrm{t}\end{array}$ \\
\hline $\begin{array}{l}\text { Jahmane and } \\
\text { Gaies (2020) }\end{array}$ & France & $\begin{array}{l}\text { ESG score } \\
\text { ASSET } 4\end{array}$ & $\begin{array}{l}\text {-ROA } \\
\text {-ROE } \\
\text {-Tobin's Q }\end{array}$ & $\sqrt{ }$ & & $\begin{array}{l}\text { Financial } \\
\text { instability }\end{array}$ \\
\hline $\begin{array}{l}\text { Jha and } \\
\text { Rangarajan } \\
(2020)\end{array}$ & India & ESG score & ROA & & $\sqrt{ }$ & \\
\hline $\begin{array}{l}\text { Long et al. } \\
(2020)\end{array}$ & China & RKS score & ROA & $\sqrt{ }$ & & $\begin{array}{l}\text { state } \\
\text { ownership } \\
\text { industry } \\
\text { competition }\end{array}$ \\
\hline $\begin{array}{l}\text { Nirino et al. } \\
(2020)\end{array}$ & $\begin{array}{l}\text { European } \\
\text { firms listed } \\
\text { in the } \\
\text { STOXX } \\
\text { Europe } \\
600 \text { index }\end{array}$ & ESG score & Tobin's Q & $\sqrt{ }$ & & $\begin{array}{l}\text { Intellectual } \\
\text { capital }\end{array}$ \\
\hline $\begin{array}{l}\text { Rjiba et al. } \\
(2020)\end{array}$ & $\begin{array}{l}\text { Internation } \\
\text { al }\end{array}$ & ESG score & Tobin's Q & $\sqrt{ }$ & & $\begin{array}{l}\text { Economic } \\
\text { policy } \\
\text { uncertainty }\end{array}$ \\
\hline $\begin{array}{l}\text { Sul and Lee } \\
(2020)\end{array}$ & Korea & $\begin{array}{l}\text { ESG scores } \\
\text { from the } \\
\text { Korean } \\
\text { Corporate } \\
\text { Governance } \\
\text { Service } \\
\text { (KCGS) }\end{array}$ & Tobin's Q & $\sqrt{ }$ & & \\
\hline $\begin{array}{l}\text { Wu et al. } \\
(2020)\end{array}$ & China & RKS score & ROA & $\sqrt{ }$ & & $\begin{array}{l}\text {-Financial } \\
\text { distress }\end{array}$ \\
\hline $\begin{array}{l}\text { Zhang et al. } \\
(2020)\end{array}$ & China & $\begin{array}{l}\text { ESG } \\
\text { disclosure } \\
\text { score from } \\
\text { Bloomberg } \\
\text { dataset } \\
\end{array}$ & Tobin's Q & $\sqrt{ }$ & & $\begin{array}{l}\text { green } \\
\text { innovation }\end{array}$ \\
\hline $\begin{array}{l}\text { Abdi et al } \\
(2021)\end{array}$ & $\begin{array}{l}\text { internation } \\
\text { al }\end{array}$ & $\begin{array}{l}\text { ESG score } \\
\text { ASSET } 4\end{array}$ & MTB & $\sqrt{ }$ & & $\begin{array}{l}\text { Firm size } \\
\text { Firm age }\end{array}$ \\
\hline $\begin{array}{l}\text { Chouaibi and } \\
\text { Chouaibi } \\
(2021)\end{array}$ & $\begin{array}{l}\text { Internation } \\
\text { al }\end{array}$ & $\begin{array}{l}\text { ESG score } \\
\text { ASSET } 4\end{array}$ & MTB & $\sqrt{ }$ & & $\begin{array}{l}\text { green } \\
\text { innovation }\end{array}$ \\
\hline $\begin{array}{l}\text { Cordeiro et } \\
\text { al. }(2021)\end{array}$ & India & CSR ratings & -ROA & & $\begin{array}{l}\text { U- } \\
\text { shaped }\end{array}$ & family firms \\
\hline
\end{tabular}




\begin{tabular}{|c|c|c|c|c|c|c|}
\hline & & & $\begin{array}{l}\text {-Annualized } \\
\text { stock return }\end{array}$ & & $\begin{array}{l}\text { curviline } \\
\text { ar }\end{array}$ & \\
\hline $\begin{array}{l}\text { Fourati and } \\
\text { Dammak } \\
(2021)\end{array}$ & $\begin{array}{l}\text { Internation } \\
\text { al }\end{array}$ & $\begin{array}{l}\text { Economic, } \\
\text { environmenta } \\
1 \text {, social and } \\
\text { governance } \\
\text { scores }\end{array}$ & $\begin{array}{l}\text {-ROA } \\
\text {-ROE }\end{array}$ & $\sqrt{ }$ & & $\begin{array}{l}\text { Corporate } \\
\text { reputation }\end{array}$ \\
\hline Park (2021) & Korea & KEJI Index & ROA & $\sqrt{ }$ & & \\
\hline $\begin{array}{l}\text { Pekovic and } \\
\text { Vogt (2021) }\end{array}$ & $\begin{array}{l}\text { Internation } \\
\text { al }\end{array}$ & $\begin{array}{l}\text { CSR score } \\
\text { from MSCI } \\
\text { ESG (KLD) } \\
\text { Stats }\end{array}$ & Tobin's Q & $\sqrt{ }$ & & $\begin{array}{l}\text { board size } \\
\text { gender } \\
\text { diversity an } \\
\text { d ownership } \\
\text { concentratio } \\
\text { n }\end{array}$ \\
\hline $\begin{array}{l}\text { Ramzan et al. } \\
(2021)\end{array}$ & Pakistan & $\begin{array}{l}\text { The natural } \\
\text { log of the } \\
\text { actual } \\
\text { amount spent } \\
\text { on CSR } \\
\text { activities }\end{array}$ & $\begin{array}{l}\text {-ROA } \\
\text {-ROE } \\
\text {-Earnings Per } \\
\text { Share } \\
\text {-Net Profit } \\
\text { Margin }\end{array}$ & $\sqrt{ }$ & & \\
\hline $\begin{array}{l}\text { Ratajczak } \\
\text { (2021) }\end{array}$ & $\begin{array}{l}\text { Internation } \\
\text { al }\end{array}$ & $\begin{array}{l}\text { Two CSR } \\
\text { dimensions " } \\
\text { (environment } \\
\text { al and social) }\end{array}$ & $\begin{array}{l}\text { Price to book } \\
\text { value }\end{array}$ & $\sqrt{ }$ & & $\begin{array}{l}\text { Natural and social } \\
\text { resources }\end{array}$ \\
\hline $\begin{array}{l}\text { Rossi et al. } \\
\text { (2021) }\end{array}$ & $\begin{array}{l}\text { European } \\
\text { countries }\end{array}$ & $\begin{array}{l}\text { ESG score } \\
\text { ASSET } 4\end{array}$ & $\begin{array}{l}\text {-ROA } \\
\text {-ROE } \\
\text {-Tobin's Q } \\
\text {-MTBV }\end{array}$ & $\sqrt{ }$ & & $\begin{array}{l}\text { Board } \\
\text { characteristi } \\
\text { cs (Duality, } \\
\text { Independen } \\
\text { ce, Board } \\
\text { size) }\end{array}$ \\
\hline $\begin{array}{l}\text { Thuy et al. } \\
\text { (2021) }\end{array}$ & Vietnam & $\begin{array}{l}\text { content } \\
\text { analysis } \\
\text { method }\end{array}$ & ROA & $\sqrt{ }$ & & $\begin{array}{l}\text { Financial } \\
\text { statement } \\
\text { comparabili } \\
\text { ty }\end{array}$ \\
\hline
\end{tabular}

\section{Metrics of Profitability and Firm Value}

Largely, it is complicated to establish a complete definition and clear methodology for firm performance. However, scholars have suggested several definitions and methodologies. Zahay and Griffen (2004) defined firm performance as the firm ability to achieve its goals while meeting the needs of its stakeholders. Ramezan et al. (2013) referred to firm performance as the ability to effectively gather and manage their assets in terms of human, financial or physical assets in order to accomplish their desired goals.

Firm performance can be measured by financial or non-financial indicators. In the literature, it is agreed that the use of the term firm performance is generally related to the 
financial aspect. Indeed, several authors use it interchangeably with profitability and market value of the company. Only a few studies established the difference between profitability and firm value. The two terms seem to overlap. Profitability is generally measured by accounting measures (ROA, ROE, Net profit, ROS, Return on capital employed). These same measures are used in other studies to measure the financial performance. Some authors measure market value mainly by stock return and private equity, the market to book ratio and the Tobin's Q. Other authors used questionnaires to construct a performance measure based on the items related to -earning per share -firm growth -net earnings per employee. These measures are generally more personalized and adapted to the studied industry. For example, in their study of luxury Andalusian hotels, González-Rodríguez et al. (2019) measure the performance of the hotel by revenue per available room. Some other authors consider leverage (total equity / total debt) as a measure of financial performance. The most recent studies focus on stock returns and future financial performance (Jeong et al. 2018) and abnormal returns (Landi and Sciaretti, 2019; Jeong et al. 2018; Cao et al. 2019) to consider such a measure.

In our literature review, the most used measure of firm performance is Tobin's Q. This measure is also used as firm value measure. The choice of this measure is based on three motivations. First, it brings together stock and accounting data and reflects market expectations for future profits. Unlike accounting measures that are based on past events, Tobin's Q focuses on expectations for future performance. Second, compared to accounting measures such as $\mathrm{ROE} / \mathrm{ROA} / \mathrm{ROI}$, it is less sensitive to management's choices regarding the principles of asset valuation. Third, the accounting measures are subject to a lot of criticism. In particular, the accounting standards still differ from one country to another. Therefore, these measurements do not allow for a reliable international comparison. On the other hand, questionable practices and accounting fraud can be practiced so that the evaluation of the performance is no longer credible.

\section{CSR Metrics}

We can suggest that the concept of CSR is self-defined; firms are encouraged to behave in a "socially responsible" way. However, such simplistic clarity is illusory. The Commission of the European Communities has tried on several occasions to define corporate social responsibility. In 2001, CSR was defined as “A concept by which companies integrate social and environmental concerns into their business operations and into their interactions with their stakeholders on a voluntary basis" (Commission of the European Communities, 2001). 
Corporate social responsibility is therefore defined as essentially a concept according to which companies voluntarily decide to contribute to a better society and to a cleaner environment. Later, the Commission of the European Communities extended the previous definition to include the economic dimension. In 2002, CSR was defined as "Corporate social responsibility concerns companies with responsibilities and taking measures beyond their legal obligations and their economic and commercial objectives. These broader responsibilities cover a range of areas, but are often summarized as social and environmental. This can be summarized as the triple performance approach: that is, economic, social and environmental" (Commission of the European Communities, 2002). A broader definition has been proposed by the United Nations: "CSR can be defined as the global contribution of companies to sustainable development". In a conceptual study, Jones (1980) defines CSR as "The notion that companies have an obligation to the constituent groups of society other than shareholders and beyond what is prescribed by law or the union contract..." Another definition that highlights stakeholders was proposed by Van Marrewijk (2003) "In general, corporate sustainability and CSR refer to corporate activities - voluntary by definition - demonstrating the inclusion of social concerns and environmental issues in business operations and in interactions with stakeholders". McWilliams and Siegel (2001) add that "CSR is a set of actions that seem to promote a social good, beyond the interests of the company and what is required by law".

In short, there is approval that social responsibility consists in taking into account the consequences of firm's activity not only on the basis of its financial performance but also on its social and environmental performance, as well as on its relations with the various stakeholders and the objectives of the sustainable development, either on mandatory (regulatory) or on voluntary bases. Although the concept of CSR is widely defined and debated in literature and practice, there is still no consensus on its measurement. Indeed, various measurements of CSR were used in the literature. Some authors have used a dummy variable to measure CSR (Bocquet et al. 2017; Miller et al. 2018). This measure does not allow reflecting and analyzing in detail the relationship between CSR and firm performance. In fact, companies engage in CSR at different levels. A dummy variable does not reveal the company's commitment. Other authors, therefore, have developed scores by themselves. These scores are personalized in relation to the country and the firms studied. The scores are constructed either from a firm-by-firm questionnaire or by content analytic method based on annual reports. The items used in these scores are generally extracted from the global reporting initiatives. Most authors opted for pre-calculated scores issued by extra-financial rating agencies. 
It is interesting to note that extra-financial rating agencies provide ratings that allow better evaluation of companies according to the so-called ESG (Environmental, Social and Governance) criteria which form the three most relevant pillars in the extra financial analysis. The most known extra-financial rating agency is MSCI ESG Research. It is made up of the absorption of several historical ESG research providers. Thus, it bought RiskMetrics in March 2010, which itself bought ISS (Institutional Shareholder Services) in 2007, Innovest Strategic Value Advisors and Kinder, Lydenberg, and Domini (KLD) in 2009. The rating aims to assess the risks and companies' environmental, social and governance (ESG) opportunities on the basis of an "AAA" to "CCC" scale depending on their exposure to industry-specific ESG risks and their ability to manage these risks in relation to their peers. The MSCI ratings have been accessible via the Factset database since 2012.

In partnership with financial and ethical organizations, the Vigeo Eiris agency generates indices and ratings to rank the best performing companies in terms of CSR. The Vigeo agency notes the performance of companies on the basis of 38 ESG issues grouped into six areas: Environment, Social commitment, Human rights, Human resources, corporate governance, and behavior on the markets. Ratings in terms of absolute performance range from 0 to 100 . This score is used to assign a performance rating from - - to ++ . Sustainalytics which is an agency specializing in ESG research and rating, assesses the ESG performance of companies using 70 specific indicators weighted according to the sector of activity. It assesses CSR performance using the best of the sector approach, called "Best in class" or even "Best of Sector", making it possible to identify the leaders and red lanterns of each sector of activity. This agency also provides analyses on the firms involvement degree in controversial sectors (tobacco, alcohol, weapons, fur, gambling, nuclear) as well as the assessment of the exposure of States to the main ESG risks (natural risks, their level of human development, respect for freedom such as freedom of the press, etc.). From 2009, Thomson Reuters acquired the provider of ratings for the Swiss ESG practices Asset4. Thomson Reuters identifies 10 categories across the three ESG dimensions for non-financial ratings. More specifically, the use of resources, emissions and innovation from an environmental perspective; workforce, human rights, community and product responsibility under the social and management dimension, shareholder rights and CSR visions and strategies under the governance dimension. Thomson Reuters also provides ESG controversy scores that reflect poor CSR practices. Regarding ESG scores, authors frequently use each indicator in its own right to test the component that has an impact on the financial performance. 


\section{CSR, Profitability and Firm Value Link: Empirical Literature}

Although the relationship between CSR and financial performance was widely examined empirically, the results are still inconclusive. A number of authors have found a negative or insignificant effect (Chen et al. 2018; Duque-Grisales and Aguilera-Caracuel, 2019). This negative association is explained by the waste of precious resources by an overinvestment in CSR. As a result, companies can improve their performance and reduce their risk by reducing CSR investments. On the other hand, the negative association can be explained by mandatory CSR reporting in several contexts e.g. Chinese firms are required to report CSR information. Mandatory CSR reporting alters the firm behavior and generates positive externalities at the expense of shareholders (Chen et al. 2018). Moreover, a negative or insignificant relationship is explained by the time horizon of the analysis. Yang (2016) argues that there may be a shortterm negative relationship, but the long-term positive relationship will eventually dominate. Other studies confirm that a socially responsible behavior has a positive impact on a firm profitability and value (Jeong et al. 2018; Chuang and Huang, 2018; Ghaderi et al. 2019; González-Rodríguez et al. 2019; Chung et al. 2018; Kim et al. 2018; Sroufe and GopalakrishnaRemani, 2019).

These confused results in the recent literature can be attributed to several factors. First, the different measures of CSR and firm profitability and value may be at the origin of this misconception. For German companies, Velte (2017) notes that ESG performance exerts a positive impact on ROA but have no impact on Tobin's Q. Likewise, the multidimensionality of the concept of CSR may be behind the mixed results. In fact, the different CSR components can have different effects on firm performance. Each component has a distinctive effect on performance. If we combine two components with contradictory effects, the result of the impact of the overall CSR measure might be insignificant or even take the sign of the component that has the greatest impact in absolute value. For example, Velte (2017) notes that environmental and social performances have weaker impact on financial performance compared to governance performance. In addition, CSR scores assess negative and positive practices. The combination of these components can generate an insignificant result. The separation of positive and negative practices is relevant. Moreover, several recent studies have separated the two aspects in the study of the CSR-firm value relationship. Almost all of these studies found that ESG good practices increase the firm value and that ESG bad practices decrease it (Fatemi et al. 2018; Price and Sun, 2017). The disclosure of information on ESG practices (good and bad) lowers the value of the company due to the nuanced picture that emerges once the 
disclosure of good practices interacts with the disclosure of bad practices. In case the company has a high social performance, a revelation of high ESG information weakens the positive valuation effect of good practices. One possible explanation for this conclusion is that markets can interpret an increased disclosure as the company's attempt to justify overinvestment in ESG activities. On the other hand, disclosure weakens the negative effects of bad ESG practices, because disclosures help companies justify their irresponsible behavior by explaining to investors the relevance of their ESG operations and policies or because they persuade investors that they have made credible commitments to overcome these bad practices. We may note that the distinction between CSR strengths and concerns is a major distinction in various social or sustainability indices. We may also note the recent literature tendency to emphasize this distinction and the relationship between these different components on the firm value (Youn et al. 2015; Price and Sun, 2017; Fatemi et al. 2018; Al-Hadi et al. 2019; Kim et al. 2018; Walker et al. 2019).

Second, the study of different contexts and different periods can be the origin of confusing results regarding the relationship between CSR and firm value. For some contexts, laws requiring the disclosure of the social and environmental consequences of firm activities in annual reports are introduced. In France, article 116 of the law on the New Economic Regulations (NRE) asserts that listed companies must communicate information relating to their social and environmental impacts in their annual reports. It is important to note here that this law does not provide for any sanction or judgment for the activities of companies. By this article, the law does not prescribe social rules to be implemented, new polluting emission thresholds, or the use of renewable energies. Thus, this law does not create an obligation "to do" but an obligation "to say". Indeed, it forces the company to publish information on its CSR; the only action constraint is therefore the collection of information and the drafting of the report in question. For other contexts, CSR is not well established, and companies are not required to invest in CSR by law. It should be noted that over $20 \%$ of under review studies were rather interested in the American context. Recent studies have extended the analysis to different developed and developing countries.

Third, recent studies highlighted the importance of the industry to which the company belongs. Indeed, industry can determine the relationship between CSR, firm value and profitability. Blasi et al. (2018), show that companies, operating in the Oil and Gas sectors, are investing more in all CSR areas. They found that the CSR engagement measured by an ESG score extracted from the KLD database increases financial performance and reduces financial 
risks. After comparing the results of manufacturing and non-manufacturing industry corporations, Chung et al. (2018) show that Soundness, Fairness, Consumer, Environment, Employee, and Development have significant effects on Tobin's Q. However, the Social score has no significant effect for manufacturing firms. In contrast, the results for corporations in other industries show that only Social, Employee, and Development scores have significant relations with Tobin's Q, and that Soundness, Fairness, Consumer, and Environment have no significant effects. Another trend in the literature is the study of the CSR firm value relationship for banks (Maqbool and Zameer, 2018; Miller et al. 2018) and firms belonging to the tourism sector (González-Rodríguez et al. 2019; Ghaderi et al. 2019; Youn et al. 2015). The separate study of banks is explained by the fact that studies related to corporate governance excluded financial institutions from the analysis due to the difference in their accounting measures and governance system compared to other sectors.

Finally, recent literature reveals channels through which CSR impacts firm value and profitability. In fact, corporate social responsibility can create additional benefits for the company that may affect its value and profitability. On the one hand, several papers have sought moderators of this relationship. For the French context, Nekhili et al. (2017) reveal the moderating role of family property in the relationship between the disclosure of information on CSR and the financial performance assessed by Tobin's Q. In the same context, Nekhili et al. (2019) suggest that employee directors in the board of directors moderate this relationship. For the U.S. listed firms, Fatemi et al. (2018) reveal that ESG disclosure plays a crucial moderating role by mitigating the negative effect of concerns and attenuating the positive effect of strengths. In their turn, Youn et al., (2015) note that firm size moderates the effect of CSR strengths on financial performance but does not moderate the effect of CSR concerns on financial performance in the U.S. restaurant context. A study on the impact of the different ESG dimensions on the ROA, conducted by Duque-Grisales and Aguilera-Caracuel (2019), notes that the financial slack and geographic international diversification play a moderating role in the relationship. However, Anser et al. (2018) failed to reveal a moderating effect of innovation in the relationship between CSR and firm profitability measured by ROA.

On the other hand, most of the recent studies tend to focus on finding out mediators for the CSR-business performance relationship. In fact, Agyemang and Ansong (2017) specify that access to capital and firm reputation mediate the CSR and firm performance relationship. In fact, CSR exerts a direct effect on firm reputation and its access to capital which in turn improves the firm financial performance. Similar results were revealed by Saeidi et al. (2015). 
The authors show an indirect role of CSR in promoting firm performance through enhancing reputation and competitive advantage while improving the customer satisfaction level. According to Martinez-Conesa et al. (2017), the firm's innovation contributes to explaining the relationship between CSR activities and financial performance.

Furthermore, several other studies have shown that companies with a higher CSR commitment face fewer constraints (Al-Hadi et al. 2019), reduce their equity and corporate debt cost (El Ghoul et al. 2017). Recently, Cui et al. (2018) have noted a negative effect of the CSR on the information asymmetry in American companies. Indeed, socially efficient companies are more closely followed by analysts and receive more favorable recommendations from them (Ioannou and Serafeim, 2015), which leads to greater accuracy in the analysts' forecasts (Dhaliwal and al. 2012). In addition, CSR improves communication with shareholders on financial matters and encourages more effective corporate governance and greater corporate value (Jo and Harjoto, 2012). These studies can be considered as the starting point for a deeper research into other mediating and moderating effects of the CSR-firm performance relationship.

\section{Conclusion}

In this study, we presented a literature review of the recent studies that have investigated the relationship between corporate social responsibility and firm performance. We showed that studies on this relationship have evolved and covered a wide variety of topics such as the effect of the CSR different components on financial performance, distinction between strengths and concerns in the analysis, dependence on the industry relationship to which the company belongs and the possible mediators and moderators of the relationship. In spite of this growing body of literature on this relationship, there are still many questions that have not received adequate replies yet. First, given the mixed results of the relationship between CSR and firm value, it would be interesting to seek an optimal CSR level and the shape of the relationship. It means that investing below or above this optimal level can have a negative effect on financial performance. From an agency perspective, managers tend to over invest in CSR and shareholders set up mechanisms to control the intensity of investment in CSR so as not to negatively affect the company value. At this point, it would also be relevant to study the impact of the ownership structure on the optimal CSR level. Second, another interesting research path could be the examination of the reverse causality in the CSR- Firm performance relationship. In fact, the studies on the financial performance impact on CSR are rather scarce. Third, It 
would be interesting to intensify the future research following the steps of (Liao et al. 2018; Hawn and Ioannou, 2016; Walker et al. 2019; Bajic and Yurtoglu, 2018; Aouadi and Marsat, 2018; García-Sánchez and Martínez-Ferrero, 2019) how adopted a meta-analysis research and apply it to establish an international comparison between companies from different countries and carry out an analysis by industry to identify the dependence of the relationship on the context analyzed and the characteristics inherent in the meaning and intensity of the impact. Fourth, in the study of the impact of the different components of the CSR index on the value of the company, some authors made the difference between strengths and concerns. On the other hand, Price and Sun (2017) made the exception of studying the intensity and sustainability of the impact of strengths which reflect both responsibility and concerns. These authors concluded that corporate irresponsibility has a greater and longer lasting impact than corporate social responsibility on the value and risk of companies. One interesting question in this context could address the kind of mechanism through which irresponsibility is more influencing than responsibility on the firm value. Finally, the underlying mechanisms through which CSR affects the firm value and profitability are not well understood. Several papers in our suggested literature review assumed that the relationship is not direct and tried to find channels through which CSR affects the firm value. We may note that most of the mediators such as the reputation, competitive advantage and innovation performance of the company are intangible. We can conclude that this relationship has attracted a great deal of research and will still attract a great deal of research ever in the future.

\section{References}

Abdi, Y., Li, X., \& Càmara-Turull, X. (2021). Exploring the impact of sustainability (ESG) disclosure on firm value and financial performance (FP) in airline industry: the moderating role of size and age. Environment, Development and Sustainability, 1-28.

Agyemang, O. S., \& Ansong, A. (2017). Corporate social responsibility and firm performance of Ghanaian SMEs: Mediating role of access to capital and firm reputation. Journal of Global Responsibility, 8(1), 47-62

Akben-Selcuk, E. (2019). Corporate social responsibility and financial performance: The moderating role of ownership concentration in Turkey. Sustainability, 11(13), 3643.

Al-Hadi, A., Chatterjee, B., Yaftian, A., Taylor, G., \& Monzur Hasan, M. (2019). Corporate social responsibility performance, financial distress and firm life cycle: evidence from Australia. Accounting \& Finance, 59(2), 961-989.

Anser, M. K., Zhang, Z., \& Kanwal, L. (2018). Moderating effect of innovation on corporate social responsibility and firm performance in realm of sustainable development. Corporate Social Responsibility and Environmental Management, 25(5), 799-806.

Aouadi, A., \& Marsat, S. (2018). Do ESG controversies matter for firm value? Evidence from international data. Journal of Business Ethics, 151(4), 1027-1047. 
Atan, R., Alam, M. M., Said, J., \& Zamri, M. (2018). The impacts of environmental, social, and governance factors on firm performance. Management of Environmental Quality: An International Journal, 29(2), 182-194.

Awaysheh, A., Heron, R. A., Perry, T., \& Wilson, J. I. (2020). On the relation between corporate social responsibility and financial performance. Strategic Management Journal, 41(6), 965-987.

Bai, X., \& Chang, J. (2015). Corporate social responsibility and firm performance: The mediating role of marketing competence and the moderating role of market environment. Asia Pacific Journal of Management, 32(2), 505-530.

Bajic, S., \& Yurtoglu, B. (2018). Which aspects of CSR predict firm market value? . Journal of Capital Markets Studies, 2(1), 50-69.

Bardos, K. S., Ertugrul, M., \& Gao, L. S. (2020). Corporate social responsibility, product market perception, and firm value. Journal of Corporate Finance, 62, 101588.

Barney, J. (1991). Firm Resources and Sustained Competitive Advantage. Journal of Management, 17(1), 99-120.

Benlemlih, M. (2017). Corporate social responsibility and firm financing decisions: A literature review. Journal of Multinational Financial Management, 42(December), 1-10.

Bing, T., \& Li, M. (2019). Does CSR Signal the Firm Value? Evidence from China. Sustainability, 11(15), 1-22.

Blasi, S., Caporin, M., \& Fontini, F. (2018). A Multidimensional Analysis of the Relationship Between Corporate Social Responsibility and Firms' Economic Performance. Ecological Economics, 147(May), 218-229.

Bocquet, R., Le Bas, C., Mothe, C., \& Poussing, N. (2017). CSR, innovation, and firm performance in sluggish growth contexts: A firm-level empirical analysis. Journal of Business Ethics, 146(1), 241-254.

Bowman, C., \& Ambrosini, V. (2003). How the Resource-Based and the Dynamic Capability Views of the Firm Inform Corporate-Level Strategy. British journal of management, 14(4), 289-303.

Broadstock, D. C., Managi, S., Matousek, R., \& Tzeremes, N. G. (2019). Does doing "good" always translate into doing "well"? An eco-efficiency perspective. Business Strategy and the Environment, 28(6), 1199-1217.

Buchanan, B., Cao, C. X., \& Chen, C. (2018). Corporate social responsibility, firm value, and influential institutional ownership. Journal of Corporate Finance, 52(October), 73-95.

Cao, R. Q., Schniederjans, D. G., Gu, V. C., \& Schniederjans, M. J. (2019). Exploring the relationship between corporate responsibility and firm performance from a social media perspective. Social Responsibility Journal, 15(3), 296-317.

Chang, K., Shim, H., \& Yi, T. D. (2019). Corporate social responsibility, media freedom, and firm value. Finance Research Letters, 30, 1-7.

Chen, Y. C., Hung, M., \& Wang, Y. (2018). The effect of mandatory CSR disclosure on firm profitability and social externalities: Evidence from China. Journal of Accounting and Economics, 65(1), 169-190.

Cherian, J., Umar, M., Thu, P. A., Nguyen-Trang, T., Sial, M. S., \& Khuong, N. V. (2019). Does corporate social responsibility affect the financial performance of the manufacturing sector? Evidence from an emerging economy. Sustainability, 11(4), 1182.

Choongo, P. (2017). A longitudinal study of the impact of corporate social responsibility on firm performance in SMEs in Zambia. Sustainability, 9(8), 1300-1318.

Chouaibi, S.,\& Chouaibi, J. (2021). Social and ethical practices and firm value: the moderating effect of green innovation: evidence from international ESG data. International Journal of Ethics and Systems, 37 (3), 442-465. 
Chuang, S. P., \& Huang, S. J. (2018). The effect of environmental corporate social responsibility on environmental performance and business competitiveness: The mediation of green information technology capital. Journal of Business Ethics, 150(4), 991-1009.

Chung, C. Y., Jung, S., \& Young, J. (2018). Do CSR activities increase firm value? Evidence from the Korean market. Sustainability, 10(9), 3164.

Clarkson, M. E. (1995). A stakeholder framework for analyzing and evaluating corporate social performance. Academy of management review, 20(1), 92-117.

Cordeiro, J. J., Galeazzo, A., \& Shaw, T. S. (2021). The CSR-CFP relationship in the presence of institutional voids and the moderating role of family ownership. Asian Business \& Management, 1-27

Cornell, B., \& Shapiro, A. C. (1987). Corporate stakeholders and corporate finance. Financial management, 16(1), 5-14.

Crifo, P., Diaye, M. A., \& Pekovic, S. (2016). CSR related management practices and Firm Performance: An Empirical Analysis of the Quantity-Quality Trade-off on French Data. International Journal of Production Economics, 171(3), 405-416.

Cui, J., Jo, H., \& Na, H. (2018). Does corporate social responsibility affect information asymmetry? Journal of Business Ethics, 148(3), 549-572.

D'Amato, A., \& Falivena, C. (2020). Corporate social responsibility and firm value: Do firm size and age matter? Empirical evidence from European listed companies. Corporate Social Responsibility and Environmental Management, 27(2), 909-924.

Dhaliwal, D. S., Radhakrishnan, S., Tsang, A., \& Yang, Y. G. (2012). Nonfinancial disclosure and analyst forecast accuracy: International evidence on corporate social responsibility disclosure. The Accounting Review, 87(3), 723-759.

Donaldson, T., \& Preston, L. E. (1995). The stakeholder theory of the corporation: Concepts, evidence, and implications. Academy of management Review, 20(1), 65-91.

Duque-Grisales, E., \& Aguilera-Caracuel, J. (2019). Environmental, social and governance (ESG) scores and financial performance of Multilatinas: Moderating effects of geographic international diversification and financial slack. Journal of Business Ethics, $1-20$.

Eccles, R. G., \& Serafeim, G. (2013). A tale of two stories: Sustainability and the quarterly earnings call. Journal of Applied Corporate Finance, 25(3), 8-19.

El Ghoul, S., Guedhami, O., \& Kim, Y. (2017). Country-level institutions, firm value, and the role of corporate social responsibility initiatives. Journal of International Business Studies, 48(3), 360-385.

Famiyeh, S. (2017). Corporate social responsibility and firm's performance: empirical evidence. Social Responsibility Journal, 13(2), 390-406.

Fatemi, A., Glaum, M., \& Kaiser, S. (2018). ESG performance and firm value: The moderating role of disclosure. Global Finance Journal, 38(C), 45-64.

Fombrun, C., \& Shanley, M. (1990). What's in a name? Reputation building and corporate strategy. Academy of management Journal, 33(2), 233-258.

Fourati, Y. M., \& Dammak, M. (2021). Corporate social responsibility and financial performance: International evidence of the mediating role of reputation. Corporate Social Responsibility and Environmental Management.

Franco, S., Caroli, M. G., Cappa, F., \& Del Chiappa, G. (2020). Are you good enough? CSR, quality management and corporate financial performance in the hospitality industry. International Journal of Hospitality Management, 88, 102395.

Freeman, R. E. (1984). Strategic Management: A Stakeholder Approach, Pitman series in business and public policy, Boston, 276. 
Friedman, M. (1962). The Relation Between Economic Freedom and Political Freedom. In Friedman, M. (Ed.), Capitalism and Freedom, University of Chicago Press, 7-17.

Friedman, M. (1970, September 13). The social responsibility of business is to increase profit. New York Times Magazine, 32-33.

Galant, A., \& Cadez, S. (2017). Corporate social responsibility and financial performance relationship: a review of measurement approaches. Economic research-Ekonomska istraživanja, 30(1), 676-693.

Garcia, A. S., Mendes-Da-Silva, W., \& Orsato, R. J. (2019). Corporate Sustainability, Capital Markets, and ESG Performance. In Individual Behaviors and Technologies for Financial Innovations (pp. 287-309). Springer, Cham.

García-Sánchez, I. M., \& Martínez-Ferrero, J. (2019). Chief executive officer ability, corporate social responsibility, and financial performance: The moderating role of the environment. Business strategy and the environment, 28(4), 542-555.

Ghaderi, Z., Mirzapour, M., Henderson, J. C., \& Richardson, S. (2019). Corporate social responsibility and hotel performance: A view from Tehran, Iran. Tourism Management Perspectives, 29(January), 41-47.

González-Rodríguez, M. R., Martín-Samper, R. C., Köseoglu, M. A., \& Okumus, F. (2019). Hotels' corporate social responsibility practices, organizational culture, firm reputation, and performance. Journal of Sustainable Tourism, 27(3), 398-419.

Guerrero-Villegas, J., Sierra-García, L., \& Palacios-Florencio, B. (2018). The role of sustainable development and innovation on firm performance. Corporate Social Responsibility and Environmental Management, 25(6), 1350-1362.

Harjoto, M., \& Laksmana, I. (2018). The impact of corporate social responsibility on risk taking and firm value. Journal of Business Ethics, 151(2), 353-373.

Harjoto, M., Laksmana, I., \& Lee, R. (2015). Board diversity and corporate social responsibility. Journal of Business Ethics, 132(4), 641-660.

Hart, S. L., \& Christensen, C. M. (2002). The great leap: Driving innovation from the base of the pyramid. MIT Sloan management review, 44(1), 51-56.

Hasan, I., Kobeissi, N., Liu, L., \& Wang, H. (2018). Corporate social responsibility and firm financial performance: The mediating role of productivity. Journal of Business Ethics, 149(3), 671-688.

Hawn, O., \& Ioannou, I. (2016). Mind the gap: The interplay between external and internal actions in the case of corporate social responsibility. Strategic Management Journal, $37(13), 2569-2588$.

Hill, C. W., \& Jones, T. M. (1992). Stakeholder-agency theory. Journal of management studies, 29(2), 131-154.

Hou, T. C. T. (2019). The relationship between corporate social responsibility and sustainable financial performance: Firm-level evidence from Taiwan. Corporate Social Responsibility and Environmental Management, 26(1), 19-28.

Ioannou, I., \& Serafeim, G. (2015). The impact of corporate social responsibility on investment recommendations: Analysts' perceptions and shifting institutional logics. Strategic Management Journal, 36(7), 1053-1081.

Jahmane, A., \& Gaies, B. (2020). Corporate social responsibility, financial instability and corporate financial performance: Linear, non-linear and spillover effects-The case of the CAC 40 companies. Finance Research Letters, 34, 101483.

Jensen, M. (2001). Value maximisation, stakeholder theory, and the corporate objective function. European financial management, 7(3), 297-317.

Jensen, M. C., \& Meckling, W. H. (1976). Theory of the firm: Managerial behavior, agency costs and ownership structure. Journal of financial economics, 3(4), 305-360. 
Jeong, K. H., Jeong, S. W., Lee, W. J., \& Bae, S. H. (2018). Permanency of CSR activities and firm value. Journal of Business Ethics, 152(1), 207-223.

Jha, M. K., \& Rangarajan, K. (2020). Analysis of corporate sustainability performance and corporate financial performance causal linkage in the Indian context. Asian Journal of Sustainability and Social Responsibility, 5, 1-30.

Jitmaneeroj, B. (2017). The impact of corporate social responsibility on firm value: an application of structural equation modelling. International Journal of Business Governance and Ethics, 12(4), 306-329.

Jo, H., \& Harjoto, M. A. (2012). The causal effect of corporate governance on corporate social responsibility. Journal of Business Ethics, 106(1), 53-72.

Jones, T. M. (1980). Corporate social responsibility revisited, redefined. California management review, 22(3), 59-67.

Kao, E. H., Yeh, C. C., Wang, L. H., \& Fung, H. G. (2018). The relationship between CSR and performance: Evidence in China. Pacific-Basin Finance Journal, 51(2018), 155-170.

Kim, K. H., Kim, M., \& Qian, C. (2018). Effects of corporate social responsibility on corporate financial performance: A competitive-action perspective. Journal of Management, 44(3), 1097-1118.

Kolsi, M. C., \& Attayah, O. F. (2018). Environmental policy disclosures and sustainable development: Determinants, measure and impact on firm value for ADX listed companies. Corporate Social Responsibility and Environmental Management, 25(5), 807-818.

Landi, G., \& Sciarelli, M. (2019). Towards a more ethical market: the impact of ESG rating on corporate financial performance. Social Responsibility Journal, 15(1), 11-27.

Liao, P. C., Shih, Y. N., Wu, C. L., Zhang, X. L., \& Wang, Y. (2018). Does corporate social performance pay back quickly? A longitudinal content analysis on international contractors. Journal of Cleaner Production, 170, 1328-1337.

Lins, K. V., Servaes, H., \& Tamayo, A. (2017). Social capital, trust, and firm performance: The value of corporate social responsibility during the financial crisis. The Journal of Finance, 72(4), 1785-1824.

Long, W., Li, S., Wu, H., \& Song, X. (2020). Corporate social responsibility and financial performance: The roles of government intervention and market competition. Corporate Social Responsibility and Environmental Management, 27(2), 525-541.

Maqbool, S., \& Zameer, M. N. (2018). Corporate social responsibility and financial performance: An empirical analysis of Indian banks. Future Business Journal, 4(1), 8493.

Martinez-Conesa, I., Soto-Acosta, P., \& Palacios-Manzano, M. (2017). Corporate social responsibility and its effect on innovation and firm performance: An empirical research in SMEs. Journal of Cleaner Production, 4(142), 2374-2383.

Mason, C., \& Simmons, J. (2014). Embedding corporate social responsibility in corporate governance: A stakeholder systems approach. Journal of Business Ethics, 119(1), 7786.

McWilliams, A., \& Siegel, D. (2001). Corporate social responsibility: A theory of the firm perspective. Academy of management review, 26(1), 117-127.

Miller, S. R., Eden, L., \& Li, D. (2018). CSR reputation and firm performance: A dynamic approach. Journal of Business Ethics, 1-18.

Murray, K. B., \& Montanari, J. B. (1986). Strategic management of the socially responsible firm: Integrating management and marketing theory. Academy of management review, $11(4), 815-827$. 
Murwaningsari, E. (2019). The Relationship of Corporate Governance, Corporate Social Responsibilities and Corporate Financial Performance in One Continuum. Indonesian Management and Accounting Research, 9(1), 78-98.

Nekhili, M., Boukadhaba, A., Nagati, H., \& Chtioui, T. (2019). ESG performance and market value: the moderating role of employee board representation. The International Journal of Human Resource Management, 1-27.

Nekhili, M., Nagati, H., Chtioui, T., \& Rebolledo, C. (2017). Corporate social responsibility disclosure and market value: Family versus nonfamily firms. Journal of Business Research, 77(August), 41-52.

Nirino, N., Ferraris, A., Miglietta, N., \& Invernizzi, A. C. (2020). Intellectual capital: the missing link in the corporate social responsibility-financial performance relationship. Journal of Intellectual Capital.

Park, B. J. (2021). Corporate Social and Financial Performance: The Role of Firm Life Cycle in Business Groups. Sustainability, 13(13), 7445.

Pekovic, S., \& Vogt, S. (2021). The fit between corporate social responsibility and corporate governance: the impact on a firm's financial performance. Review of Managerial Science, 15(4), 1095-1125.

Perrini, F., Russo, A., Tencati, A., \& Vurro, C. (2011). Deconstructing the relationship between corporate social and financial performance. Journal of Business Ethics, 102(1), 59-76.

Porter, M. E., \& Kramer, M. R. (2002). The competitive advantage of corporate philanthropy. Harvard business review, 80(12), 56-68.

Price, J. M., \& Sun, W. (2017). Doing good and doing bad: The impact of corporate social responsibility and irresponsibility on firm performance. Journal of Business Research, 80(November), 82-97.

Ramanathan, R. (2018). Understanding complexity: The curvilinear relationship between environmental performance and firm performance. Journal of Business Ethics, 149(2), 383-393.

Ramezan, M., Sanjaghi, M. E., \& Baly, H. R. K. (2013). Organizational change capacity and organizational performance: An empirical analysis on an innovative industry. Journal of Knowledge-based Innovation in China, 5(3), 188-212.

Ramzan, M., Amin, M., \& Abbas, M. (2021). How does corporate social responsibility affect financial performance, financial stability, and financial inclusion in the banking sector? Evidence from Pakistan. Research in International Business and Finance, 55, 101314.

Rasheed, B., Arshed, N., Malik, Z. F., \& Mahmood, M. T. (2018). Impact of corporate social responsibility on firm's performance: evidence from non-financial sector of Pakistan. Afro-Asian Journal of Finance and Accounting, 8(2), 105-122.

Ratajczak, P. (2021). The mediating role of natural and social resources in the corporate social responsibility-corporate financial performance relationship. Managerial and Decision Economics, 42(1), 100-119.

Rjiba, H., Jahmane, A., \& Abid, I. (2020). Corporate social responsibility and firm value: Guiding through economic policy uncertainty. Finance Research Letters, 35, 101553.

Rossi, M., Chouaibi, J., Chouaibi, S., Jilani, W., \& Chouaibi, Y. (2021). Does a Board Characteristic Moderate the Relationship between CSR Practices and Financial Performance? Evidence from European ESG Firms. Journal of Risk and Financial Management, 14(8), 354.

Saeidi, S. P., Sofian, S., Saeidi, P., Saeidi, S. P., \& Saaeidi, S. A. (2015). How does corporate social responsibility contribute to firm financial performance? The mediating role of competitive advantage, reputation, and customer satisfaction. Journal of business research, 68(2), 341-350. 
Sekhon, A. K., \& Kathuria, L. M. (2019). Analyzing the impact of corporate social responsibility on corporate financial performance: evidence from top Indian firms. Corporate Governance: The International Journal of Business in Society, 20 (1), 143-157.

Sheikh, S. (2018). Corporate social responsibility, product market competition, and firm value. Journal of Economics and Business, 98(C), 40-55.

Sial, M. S., Zheng, C., Khuong, N. V., Khan, T., \& Usman, M. (2018, a). Does Firm Performance Influence Corporate Social Responsibility Reporting of Chinese Listed Companies?. Sustainability, 10(7), 1-12.

Sial, M., Chunmei, Z., Khan, T., \& Nguyen, V. (2018, b). Corporate social responsibility, firm performance and the moderating effect of earnings management in Chinese firms. AsiaPacific Journal of Business Administration, 10(2/3), 184-199.

Siueia, T. T., Wang, J., \& Deladem, T. G. (2019). Corporate Social Responsibility and financial performance: A comparative study in the Sub-Saharan Africa banking sector. Journal of Cleaner Production, 226, 658-668.

Sroufe, R., \& Gopalakrishna-Remani, V. (2019). Management, social sustainability, reputation, and financial performance relationships: An empirical examination of US firms. Organization \& Environment, 32(3), 331-362.

Sul, W., \& Lee, Y. (2020). Effects of corporate social responsibility for environmental, social, and governance sectors on firm value: a comparison between consumer and industrial goods companies. European Journal of International Management, 14(5), 866-890.

Thuy, C. T. M., Khuong, N. V., Canh, N. T., \& Liem, N. T. (2021). Corporate Social Responsibility Disclosure and Financial Performance: The Mediating Role of Financial Statement Comparability. Sustainability, 13(18), 10077.

Van Marrewijk, M. (2003). Concepts and definitions of CSR and corporate sustainability: Between agency and communion. Journal of Business Ethics, 44(2-3), 95-105.

Velte, P. (2017). Does ESG performance have an impact on financial performance? Evidence from Germany. Journal of Global Responsibility, 8(2), 169-178.

Velte, P. (2019). Does CEO power moderate the link between ESG performance and financial performance? A focus on the German two-tier system. Management Research Review, 43(5), 497-520.

Walker, K., Zhang, Z., \& Ni, N. (2019). The Mirror effect: Corporate social responsibility, corporate social irresponsibility and firm performance in coordinated market economies and Liberal market economies. British Journal of Management, 30(1), 151168.

Wang, D. H. M., Chen, P. H., Yu, T. H. K., \& Hsiao, C. Y. (2015). The effects of corporate social responsibility on brand equity and firm performance. Journal of business research, 68(11), 2232-2236.

Werner, W. J. (2009). Corporate social responsibility initiatives addressing social exclusion in Bangladesh. Journal of health, population, and nutrition, 27(4), 545-562.

Wu, L., Shao, Z., Yang, C., Ding, T., \& Zhang, W. (2020). The impact of CSR and financial distress on financial performance-evidence from Chinese listed companies of the manufacturing industry. Sustainability, 12(17), 6799.

Yang, S. L. (2016). Corporate social responsibility and an enterprise's operational efficiency: considering competitor's strategies and the perspectives of long-term engagement. Quality \& Quantity, 50(6), 2553-2569.

Yoon, B., Lee, J. H., \& Byun, R. (2018). Does ESG Performance Enhance Firm Value? Evidence from Korea. Sustainability, 10(10), 1-18. 
Youn, H., Hua, N., \& Lee, S. (2015). Does size matter? Corporate social responsibility and firm performance in the restaurant industry. International Journal of Hospitality Management, 51(October), 127-134.

Yu, E. P. Y., Guo, C. Q., \& Luu, B. V. (2018). Environmental, social and governance transparency and firm value. Business Strategy and the Environment, 27(7), 987-1004.

Zahay, D., \& Griffin, A. (2004). Customer learning processes, strategy selection, and performance in business-to-business service firms. Decision Sciences, 35(2), 169-203.

Zhang, F., Qin, X., \& Liu, L. (2020). The interaction effect between ESG and green innovation and its impact on firm value from the perspective of information disclosure. Sustainability, 12(5), 1866.

Zhao, Z., Meng, F., He, Y., \& Gu, Z. (2019). The Influence of Corporate Social Responsibility on Competitive Advantage with Multiple Mediations from Social Capital and Dynamic Capabilities. Sustainability, 11(1), 1-16.

Zolotoy, L., O'Sullivan, D., \& Chen, Y. (2019). Local religious norms, corporate social responsibility, and firm value. Journal of Banking \& Finance, 100, 218-233. 